\title{
Avulsão vascular retiniana
}

\author{
Avulsed retinal vessels
}

\author{
Leonardo PérezZeni ${ }^{1}$ \\ Manuel Augusto Pereira Vilela ${ }^{2}$
}

Trabalho realizado no Curso de Especialização em Oftalmologia "Ivo Corrêa-Meyer" - Porto Alegre (RS) e na Universidade Federal de Pelotas - UFPEL - Pelotas (RS) - Brasil.

${ }^{1}$ Médico do Curso de especialização em Oftalmologia "Ivo Corrêa-Meyer" - Porto Alegre (RS) - Brasil.

${ }^{2}$ Professor-Titular-Doutor, Regente da Disciplina de Oftalmologia na Universidade Federal de Pelotas - UFPEL Pelotas (RS) - Brasil.

Endereço para correspondência: Curso de Especialização em Oftalmologia "Ivo Corrêa-Meyer" - Rua Félix da Cunha, 496 - Porto Alegre (RS) CEP 90570-070

E-mail: iicm@terra.com.br

Recebido para publicação em 10.08.2004

Versão revisada recebida em 20.05.2005

Aprovação em 30.10.2005

\section{RESUMO}

Objetivo: Descrever a evolução de uma série de casos com avulsão vascular retiniana sem descolamento tratados pelo fotocoagulação ou introflexão escleral. Métodos: Análise observacional retrospectiva. No período entre 2001-2003 foram tratados 13 olhos com avulsão sem descolamento utilizando-se o laser ou a introflexão escleral. O seguimento mínimo foi de 6 meses. Média etária de 53,6 anos. Resultados: Oito olhos foram somente fotocoagulados e 5, submetidos a introflexão. $\mathrm{O}$ sintoma mais comum foi a baixa na acuidade visual. Em 7 olhos $(53,8 \%)$ a hemorragia vítrea em quantidade variável estava presente ao diagnóstico. Destes, 3 tiveram novo episódio após os procedimentos $(42,8 \%)$. A acuidade visual final em todos os olhos foi igual ou melhor do que a inicial. Nenhuma complicação foi observada. Conclusão: Nesta série de casos com avulsão vascular sem descolamento houve evolução favorável, independente das técnicas utilizadas. A hemorragia vítrea tardia é a principal intercorrência tardia observada, mas não comprometeu os resultados.

Descritores: Vasos retinianos; Perfurações retinianas; Cirurgia a laser; Hemorragia vítrea

\section{INTRODUÇÃO}

Vasos retinianos avulsionados em associação com rupturas definem uma entidade clínica denominada "síndrome da avulsão vascular retiniana", descrita, pela primeira vez, por Clark em $1962^{(1)}$. Foi caracterizada por episódios recorrentes de hemorragia vítrea associados à presença de um "rasgo retiniano" com um vaso patente atravessando sua superfície em íntimo contato com o vítreo. Em 1971 alguns autores consideraram a entidade como uma síndrome específica em que o sangramento vítreo só seria interrompido após a oclusão do vaso avulsionado ${ }^{(2)}$.

O termo avulsão vascular denota o deslocamento segmentar de um vaso retiniano de sua posição original como consequiência de tração com formação de um rasgo retiniano de espessura variável. Na maioria dos casos, os segmentos são arteriais e localizados nas regiões equatoriais e pós-equatoriais da retina ${ }^{(1-7)}$.

Nos últimos 30 anos diversas formas de terapêutica têm sido utilizadas na tentativa de diminuir a recorrência de hemorragia vítrea: fotocoagulação com laser argônio, criopexia, "buckle" escleral, implante de balão episcleral temporário entre outras ${ }^{(1-9)}$.

O presente estudo objetiva descrever a evolução de uma série de pacientes com avulsão vascular retiniana tratados somente pela fotocoagulação com laser argônio ou, então, pela introflexão escleral, discutindo as opções utilizadas. 


\section{MÉTODOS}

Trata-se de uma análise observacional retrospectiva dos prontuários arquivados dos pacientes com diagnóstico de avulsão vascular retiniana tratados nos últimos 3 anos com seguimento mínimo de 6 meses. Os mesmos foram submetidos a exame oftalmológico completo, e a fundoscopia foi realizada com o oftalmoscópio binocular indireto e depressão escleral e pela biomicroscopia posterior (lentes "superquad" Volk ${ }^{\circledR}$ e de 3 espelhos). Casos com hemorragia vítrea densa que foram seguidos com ultra-sonografia até o momento em que o exame fundoscópico fosse possível.

Estão incluídos apenas os casos documentados com este diagnóstico, com prontuários completos, sem descolamento de retina associado ou outra doença ocular ou cirurgia prévia. Foram excluídos aqueles cuja avulsão estivesse associada a descolamento de retina. $\mathrm{O}$ desfecho funcional, intercorrências dos métodos (laser ou introflexão) e surgimento ou recorrência de hemorragia vítrea foram examinados.

Os procedimentos terapêuticos foram realizados pelo mesmo oftalmologista (MV), e a opção por um ou outro método foi discutida em todos os casos.

Durante este estudo, avaliou-se a idade, sexo, sintoma predominante, localização da avulsão, presença de hemorragia vítrea inicial ou recorrente, acuidade visual final e resultado anatômico.

A análise identificou 13 olhos que obedeciam aos critérios propostos. Destes, 8 foram submetidos a fotocoagulação com laser de argônio verde e 5 , a introflexão escleral com explante (esponja) localizado (Figuras 1, 2, 3, 4). Os principais aspectos examinados estão contidos na tabela 1. Do total, 6 casos eram do sexo masculino e 7, feminino. Idade oscilou entre 30 e 70 anos, média 53,6 anos.

\section{RESULTADOS}

Em sete casos a baixa de acuidade foi o motivo da consulta. Nos demais o achado foi sinalizado pela presença de miidopsias e fosfenas. Em sete pacientes $(53,8 \%)$ a hemorragia vítrea estava presente ao diagnóstico. Todos tinham descolamento do vítreo posterior. A acuidade ao final de 6 meses estava igual, ou melhor do que a inicial, em todos os olhos. Três olhos tiveram recorrência na hemorragia vítrea $(42,8 \%)$, destes 2 permaneceram com os níveis de acuidade estáveis em relação à consulta inicial. Apenas um olho que não tinha apresentado hemorragia vítrea ao diagnóstico teve sangramento no seguimento $(1 / 6,16,6 \%)$. No total a hemorragia vítrea tardia ocorreu em 4 olhos $(30,76 \%)$.

\section{DISCUSSÃO}

A presença de um segmento vascular avulsionado em associação com uma ruptura retiniana representa uma entida- de clínica específica de difícil manejo, devido à recorrência de hemorragias na cavidade vítrea. Nas últimas décadas, foram realizados, em pequena escala, estudos na busca do método terapêutico mais efetivo na tentativa de prevenir os episódios hemorrágicos. O objetivo comum dos tratamentos propostos consistiu em ocluir o vaso patente e aliviar a tração que o vítreo promove sobre este, evitando assim o início da hemorragia ou ressangramento ${ }^{(1-9)}$.

Em 1971, foi realizado estudo em 14 pacientes que apresentavam hemorragia vítrea associada à ruptura retiniana com avulsão vascular. Foram aplicadas três modalidades terapêuticas: fotocoagulação, criopexia e introflexão escleral mais criopexia, havendo recorrência de sangramento em todos os pacientes $\operatorname{tratados}^{(2)}$.

Acompanhamento foi iniciado em 1984 com 18 pacientes (19 olhos) durante 54 meses, realizando diversas técnicas para oclusão das rupturas incluindo diatermia, fotocoagulação e introflexão escleral. Independente do método empregado no bloqueio das rupturas retinianas, a hemorragia vítrea persistiu até a interrupção do segmento vascular destes pacientes ${ }^{(3)}$.

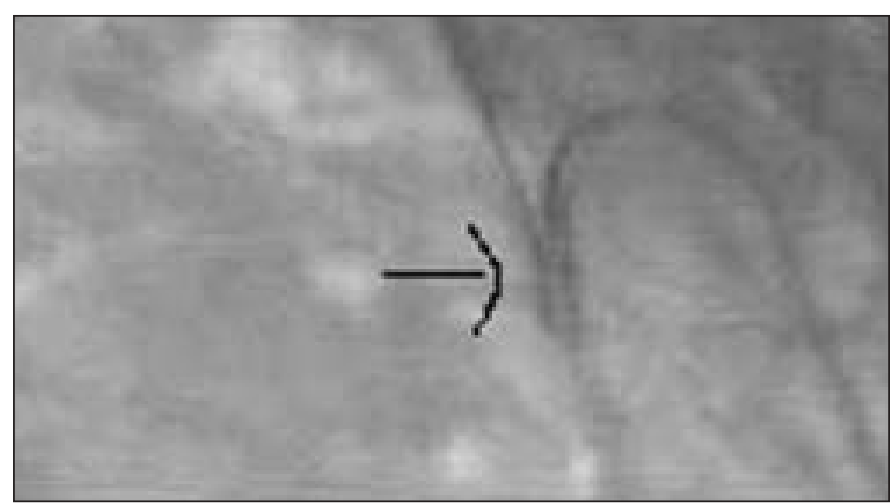

Figura 1 - A seta aponta para a avulsão, com grande projeção para o vítreo

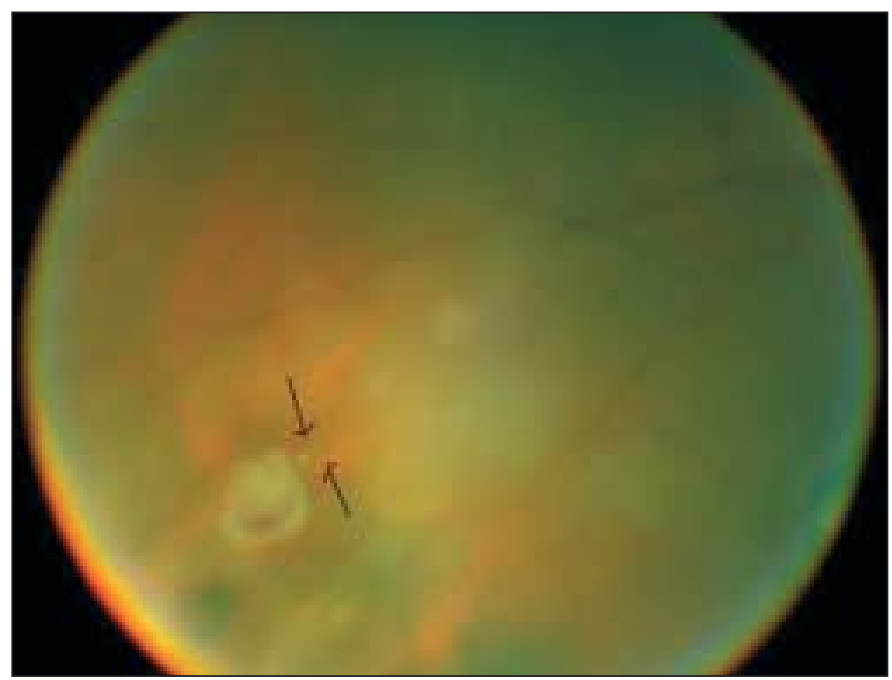

Figura 2 - 0 foco da imagem foi intencionalmente feito sobre o opérculo e as setas delimitam o percurso do vaso "em ponte" 


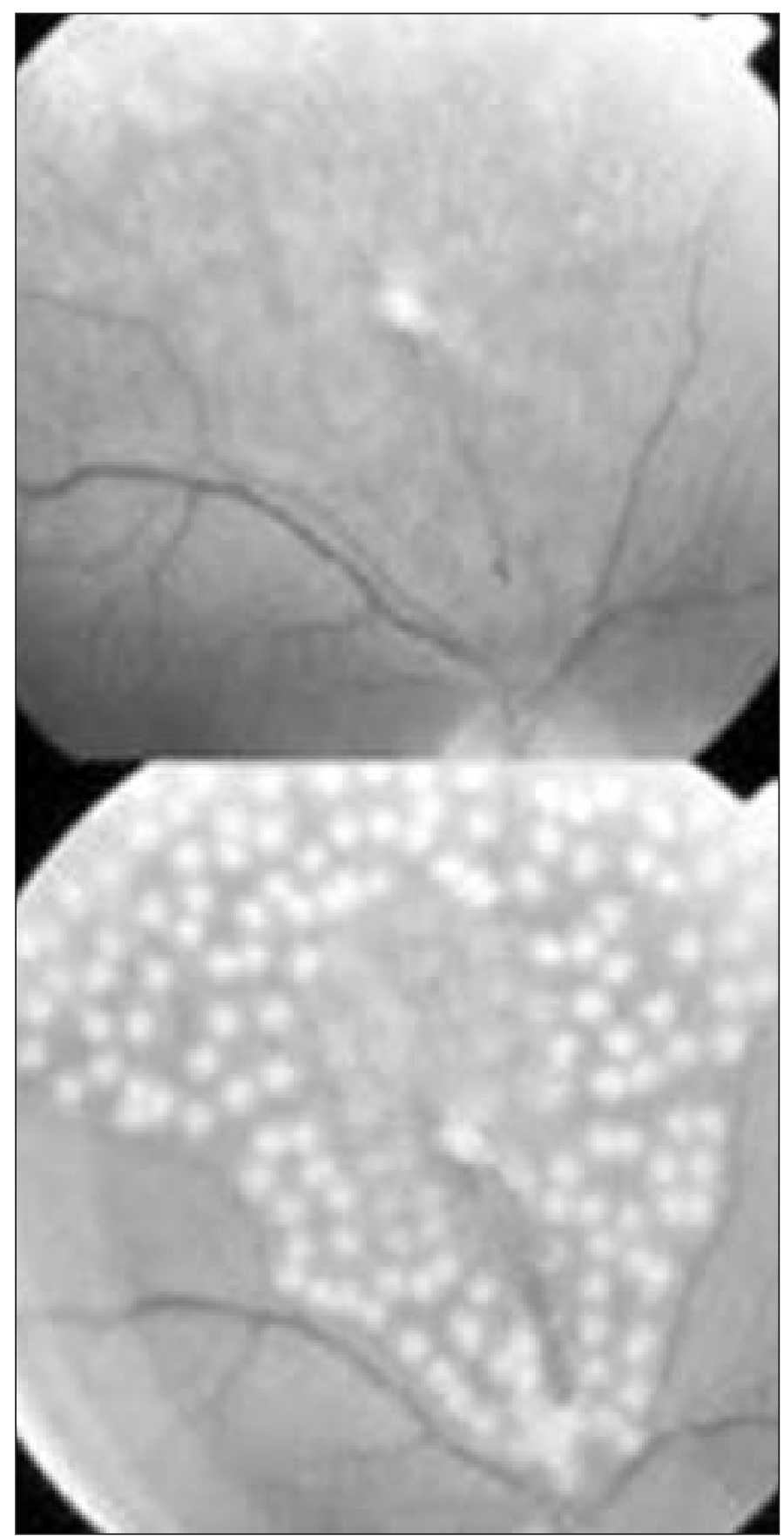

Figura 3 - Composição retinográfica mostrando na porção superior a avulsão e ponto de tração vítrea, e abaixo após a fotocoagulação

Entre 1975 e 1984 foi realizada análise de 64 pacientes com avulsão vascular retiniana comparando o uso da fotocoagulação em 30 pacientes com a introflexão escleral utilizada em 34 pacientes. Houve maior recorrência das hemorragias vítreas nos casos tratados com fotocoagulação versus introflexão escleral. No entanto esta diferença foi inválida do ponto de vista estatístico ${ }^{(4)}$.

Realizaram em 9 olhos com avulsão, a utilização de balão temporário episcleral, obtendo êxito, definido pela oclusão do

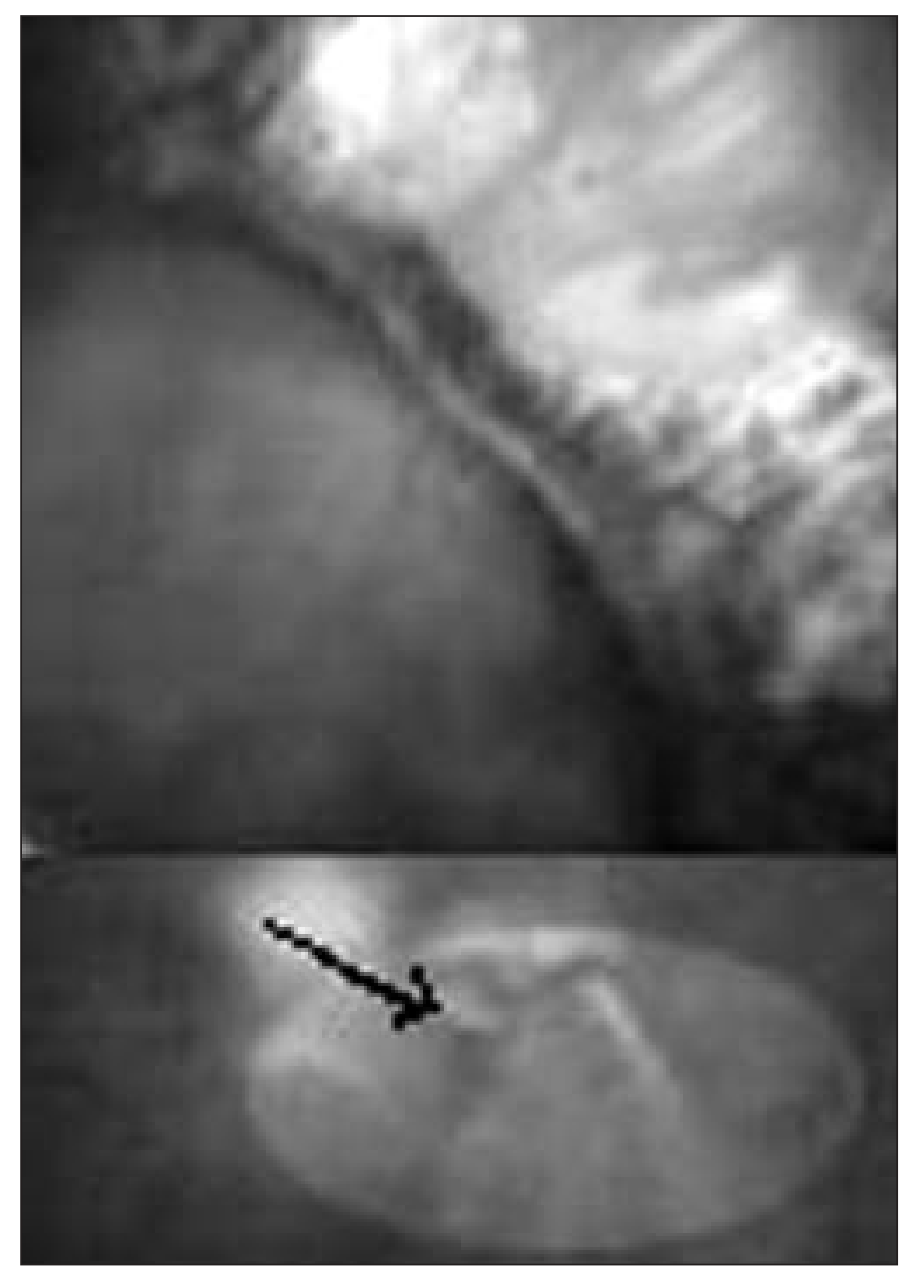

Figura 4 - Na porção inferior a seta aponta para a avulsão e ruptura. Acima a mesma área pós introflexão escleral

ramo avulsionado, em todos os casos, porém com alto índice de ressangramento vítreo ao longo do seguimento $(45 \%)^{(5)}$.

Foi sugerida a utilização da fotocoagulação com laser argônio em sessões repetidas como um método efetivo na prevenção do sangramento vítreo. Eles observaram 17 pacientes após terapia sucessiva com laser argônio, havendo recorrência de sangramento em apenas dois pacientes. No entanto não se conseguiu estabelecer uma correlação entre o seguimento destes pacientes tratados com laser com aqueles não submetidos ao tratamento por não haver grupo controle ${ }^{(6)}$.

Foi utilizado Nd:YAG laser em 3 olhos para liberar a tração vítrea de vasos "em ponte". Na média foram necessários 4403104 pulsos. Todos tiveram leve hemorragia, estancada pela compressão manual sobre o globo. No seguimento de 24 meses não foram descritas outras complicações ${ }^{(9)}$.

Nosso estudo busca realizar uma análise comparativa entre pacientes com avulsão vascular retiniana sem descolamento submetidos a fotocoagulação com laser argônio ou implante de "buckle" escleral. Os casos foram submetidos ao tratamento seguindo critérios individuais do mesmo oftalmologista, não havendo uma seleção pré-determinada. Não houve diferença do 


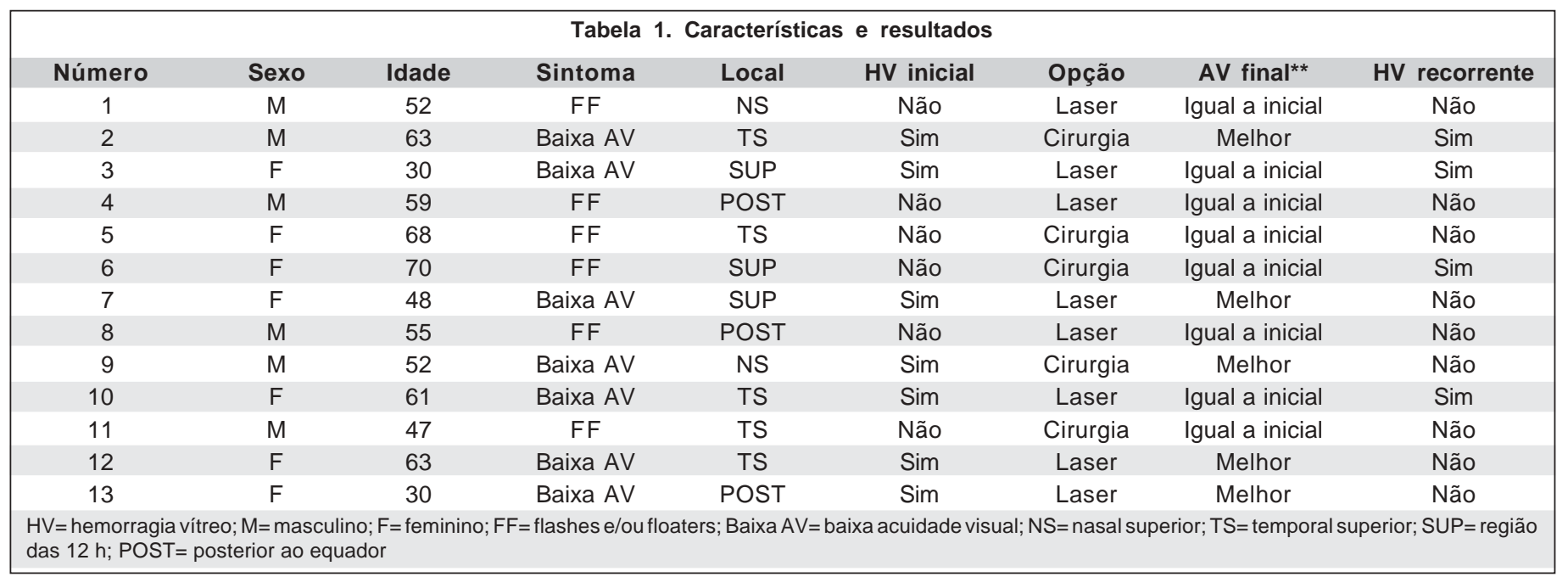

ponto de vista funcional e anatômico entre as duas técnicas realizadas. Todos os casos mantiveram ou melhoraram a acuidade. Hemorragia vítrea tardia foi observada em 4 olhos (30,7\%), destes a recorrência foi notada em 3 olhos (3/7, 42,8\%). As avulsões localizadas posteriormente ao equador foram sempre fotocoaguladas devido a melhores condições técnicas. Com nenhum dos métodos foi notada qualquer intercorrência, aspecto favorável do prognóstico que foi de encontro a todas as séries ${ }^{(1-12)}$. Como esta é uma amostra pequena, sem grupo controle, não há como definir qual seria a melhor opção, e neste aspecto reproduz as fontes aqui contidas.

É estimado que 8,5\% dos casos com descolamento regmatogênico de retina tenham sido gerados na presença de avulsão vascular e ruptura. Anatomicamente estas alterações costumam estar situadas na região pós-equatorial, com cerca de 7\% de chances de hemorragias vítreas recorrentes. Não há influência da avulsão na perspectiva de reparo anatômico ${ }^{(7-8)}$.

É importante salientar que nos casos de avulsão vascular associada à ruptura retiniana, a recorrência do sangramento vítreo decorre da viabilidade funcional do segmento avulsionado. É difícil ocluir completamente o vaso avulsionado com qualquer um dos métodos, principalmente quando existe um maior afastamento do plano retiniano. Observou-se que nos pacientes sem recidiva de hemorragia, o segmento vascular estava ocluído independente da presença de terapia ou da técnica empregada ${ }^{(2-9)}$.

\section{CONCLUSÃO}

Avulsão vascular retiniana ocorre, sob o ponto de vista conceitual, associada a descolamento posterior do vítreo, podendo provocar hemorragia vítrea recorrente e descolamento de retina. A rigor a potencialidade de ressangramento parece vinculada à patência do vaso e a continuidade dos vetores tracionais. Quando não existe descolamento de retina associado percebe-se uma semelhança nos resultados comparati- vos entre a fotocoagulação e a introflexão permanente. $\mathrm{O}$ prognóstico nestes casos é favorável.

\section{ABSTRACT}

Purpose: To describe the clinical evolution of cases with avulsed retinal vessels without retinal detachment treated with laser or scleral buckle. Methods: Retrospective analysis of cases with avulsed retinal vessels treated between 20012003. Laser photocoagulation or scleral buckle was used. The minimum follow-up was 6 months. Average age was 53.6 years. Results: Of 13 patients, 8 were photoagulated with argon laser and 5 were submitted to a scleral buckle. The most common symptom was reduction in visual acuity. Vitreous hemorrhage was present in 7 eyes (53.8\%) at diagnosis, and was the most frequent complication after treatment (30.7\%). Final visual acuity was unchanged. Conclusion: Avulsed retinal vessels have a good prognosis using laser or buckle. Late vitreous hemorrhage was the main complication.

Keywords: Retinal vessels; Retinal perforations; Laser surgery; Vitreous hemorrhage

\section{REFERÊNCIAS}

1. Clark G. The use of photocoagulator in conditions associated with retinal detachment. Trans Am Acad Ophthalmol Otolaryngol. 1962;66:59-66.

2. Robertson DM, Curtin VT, Norton EW. Avulsed retinal vessels with retinal breaks: a cause of recurrent vitreous hemorrhage. Arch Ophthalmol. 1971;85(6): 669-72.

3. De Bustros S, Welch RB. The avulsed retinal vessel syndrome and its variants. Ophthalmology. 1984;91(1):86-8.

4. Theodossiadis GP, Koutsandrea CN. Avulsed retinal vessels with and without retinal breaks - treatment and extended follow-up. Trans Ophthalmol Soc U.K. 1985;104(Pt 8):887-92.

5. Lincoff H, Kreissig I, Richard G. Treating avulsed vessels with a temporary balloon buckle. Am J Ophthalmol. 1986;101(1):90-4.

6. Folk JC, Ma C, Blodi CF, Han DP. Occlusion of bridging or avulsed retinal vessels by repeated photocoagulation. Ophthalmology. 1987;94(12):1610-3. 
7. Theodossiadis GP, Koutsandrea CN. Types of avulsed retinal vessels associated with rhegmatogenous retinal detachment: treatment and results. Graefe's Arch Clin Exp Ophthalmol. 1987;225(3):185-8.

8. Williams GA, Aaberg TM. Techniques of scleral buckling. In: Ryan SJ. Retina $2^{\text {nd }}$ ed. St Louis: Mosby; 1994. p.1979-2018.

9. Tatsui T, Ohara K, Shimizu H. Nd:YAG laser photodisruption of the vitreous traction in avulsed retinal vessel syndrome. Ophthalmic Surg. 1990;21(6): 423-7.
10. Kreissig I, Rose D, Jost B. Minimized surgery for retinal detachments with segmental buckling and nondrainage. An 11-year follow-up. Retina. 1992;12(3): 224-31.

11. Bloom SM, Brucker AJ. Laser surgery of the posterior segment. $2^{\text {nd }}$ ed. Philadelphia: Lippincott-Raven; 1997. p.351-2.

12. Schatz H. Laser treatment of fundus disease. San Anselmo: Pacific Medical Press; 1980.

\title{
XXIX Simpósio de Oftalmologia da UNIFESP
}

\section{Instituto da Visão}

\section{3 a 5 de Agosto de 2006}

\author{
LOCAL:
}

GRAN MELIÁ MOFARREJ

Al. Santos, 1437 • São Paulo (SP)

\section{TEMA: NOVAS TECNOLOGIAS}

\section{CURSOS E SIMPÓSIOS:}

- Atualização Clínica e Cirúrgica nas diversas áreas da 0 ftalmologia

- Programas de imersão no Pré-Congresso

- Atualização em Clínica Médica para ofta Imologistas

- Atualização em Medicina Celular e Células-tronco aplicadas à Oftalmologia

- Cirurgias a o vivo

- Wet Labs

- Investimentos e Finanças pessoais 